\title{
In vitro study on the role of SOX9 in trastuzumab resistance of adenocarcinoma of the esophagogastric junction
}

\author{
YINGCAI HONG $^{1}$, HUAISHENG CHEN ${ }^{1}$, ZHANPENG RAO ${ }^{1}$, \\ BIN PENG ${ }^{1}$, HONG HU ${ }^{1}$, SHAOLIN LIN ${ }^{2}$ and ZHENGLEI XU ${ }^{2}$ \\ Departments of ${ }^{1}$ Thoracic Surgery and ${ }^{2}$ Gastroenterology, Second Clinical Medical College of Jinan University, \\ Shenzhen People's Hospital, Shenzhen, Guangdong 518000, P.R. China
}

Received November 14, 2016; Accepted April 21, 2017

DOI: $10.3892 /$ etm.2018.5744

\begin{abstract}
Trastuzumab is recommended for the treatment of human epidermal growth factor receptor 2-positive adenocarcinoma of the esophagogastric junction (AEG) in combination with chemotherapy; however, drug resistance has severely affected its clinical application. The present study aimed to investigate the effect of sex determining region Y-box 9 (SOX9), a prognostic marker in adjuvant oncological settings, on AEG cell proliferation and apoptosis in the presence or absence of trastuzumab. Furthermore, the molecular mechanism underlying the role of SOX9 in trastuzumab resistance was explored. ESO26 cells were treated with various concentrations of trastuzumab, and trastuzumab induced SOX9 expression in a concentration-dependent manner, as determined by reverse transcription-quantitative polymerase chain reaction and western blotting analyses. Transfection of ESO26 cells with SOX9 small interfering RNA was conducted to knock down SOX9 expression, and the results of MTT and flow cytometry assays demonstrated that SOX9 knockdown sensitized ESO26 cells to trastuzumab by inhibiting cell proliferation and enhancing cell apoptosis. In addition, it was observed that the trastuzumab-induced phosphorylation of AKT was suppressed by SOX9 knockdown. In conclusion, the present study demonstrated that SOX9 participated in trastuzumab resistance by affecting cell proliferation and apoptosis, and indicated that SOX9 may exert its effect on trastuzumab resistance via activation of the phosphatidylinositol-3-kinase/AKT signaling pathway. This study identified a novel mechanism underlying trastuzumab resistance in vitro and may be useful in improving the efficacy of trastuzumab treatment.
\end{abstract}

Correspondence to: $\mathrm{Dr}$ Zhenglei $\mathrm{Xu}$, Department of Gastroenterology, Second Clinical Medical College of Jinan University, Shenzhen People's Hospital, 1017 North Dongmen Road, Shenzhen, Guangdong 518000, P.R. China

E-mail: xu_zhenglei@yeah.net

Key words: trastuzumab resistance, SOX9, adenocarcinoma of esophagogastric junction, PI3K/AKT signaling

\section{Introduction}

Adenocarcinoma of the esophagogastric junction (AEG) is a lethal malignancy originating from the distal esophagus and the esophagogastric junction $(1,2)$. The incidence of AEG has increased rapidly worldwide during the past two decades (3-6). The prognosis of AEG is poor due to distant metastasis at the time of diagnosis and the limited treatment options $(7,8)$. Trastuzumab, a monoclonal antibody targeting human epidermal growth factor receptor 2 (HER2), has emerged as an effective therapeutic option for AEG when combined with chemotherapy (9).

In a phase III, open-label, international, randomized controlled trial, Bang et al (9) observed that patients treated with trastuzumab plus chemotherapy had longer median follow-up and median overall survival times compared with patients treated with chemotherapy alone. The authors suggested that trastuzumab in combination with chemotherapy may be a new standard option for the first-line treatment of HER2-positive advanced gastric or gastro-esophageal junction cancer (9). International National Comprehensive Cancer Network Guidelines recommend the detection of HER2 in patients with AEG in order to guide the selection of further clinical treatment options (10). However, trastuzumab treatment is invalid for nearly half of HER2-positive patients (9), and the mechanisms of drug resistance are currently unknown. Therefore, the identification of sensitive predictive biomarkers for HER2-positive patients in whom trastuzumab treatment is invalid is important for the prognosis of advanced AEG.

Sex determining region Y-box 9 (SOX9) is a nuclear transcription factor that belongs to the group E subgroup of the SOX protein family, and its activity has been associated with several types of cancer (11-13). Chakravarty et al (14) and Riemenschnitter et al (15) suggested SOX9 to be a prognostic marker in adjuvant oncological settings. It was reported that breast cancer patients with a high SOX9 level following chemotherapy contributes to a negative prognosis and shortened overall survival (15). More importantly, the predictive efficacy of SOX9 is higher than that of other clinico-pathological parameters, such as HER2-status or pathological stage (15). However, to the best of our knowledge, the specific biological function of SOX9 in AEG has not yet been reported. 
In the present study, in vitro experiments were performed on ESO26 cells to investigate the effect of SOX9 on cell proliferation and apoptosis in the presence or absence of trastuzumab. Furthermore, whether SOX9 affects the activation of the phosphatidylinositol-3-kinase (PI3K)/AKT (also known as protein kinase B) signaling pathway, an intracellular signaling pathway that is involved in the resistance of gastric cancer to trastuzumab $(16,17)$, was explored. The results of this study may elucidate the mechanism underlying trastuzumab resistance and improve the efficacy of trastuzumab treatment.

\section{Materials and methods}

Cell culture and trastuzumab treatment. The ESO26 cell line was purchased from the European Collection of Cell Cultures (Salisbury, UK). The cells were cultured in RPMI-1640 medium (Invitrogen; Thermo Fisher Scientific, Inc., Waltham, MA, USA) supplemented with $2 \mathrm{mM}$ glutamine (Invitrogen; Thermo Fisher Scientific, Inc.) and $10 \%$ fetal bovine serum (FBS; Invitrogen; Thermo Fisher Scientific, Inc.), and maintained at $37^{\circ} \mathrm{C}$ in a humidified atmosphere of $5 \% \mathrm{CO}_{2}$. Trastuzumab was provided by Roche Pharmaceuticals Ltd. (Shanghai, China). The cells at a density of $3 \times 10^{5}$ cells/well were treated with $0,0.5,1,2$ and $4 \mathrm{mg} / \mathrm{ml}$ trastuzumab for $24 \mathrm{~h}$ in RPMI-1640 medium.

Cell transfection. Small interfering RNA (Si)-control and si-SOX9 were obtained from Zhonghong Boyuan Biological Technology Co., Ltd. (Shenzhen, China), and were transfected into ESO26 cells using Lipofectamine 2000 (Invitrogen; Thermo Fisher Scientific, Inc.) according to the manufacturer's instructions. Briefly, cells were seeded into 6-well plates at a density of $3 \times 10^{5}$ cells/well and incubated at $3^{\circ} \mathrm{C}$ overnight. At $4 \mathrm{~h}$ prior to transfection, the medium was changed to Opti-MEM (Invitrogen; Thermo Fisher Scientific, Inc.). Si-control/si-SOX9 and Lipofectamine 2000 were each diluted in $250 \mu \mathrm{l} \mathrm{Opti-MEM}$, and then mixed and incubated at $37^{\circ} \mathrm{C}$ for $20 \mathrm{~min}$. The mixture was added to each well and the cells were incubated at $37^{\circ} \mathrm{C}$ for $6 \mathrm{~h}$. After that, the medium was replaced with complete RPMI-1640 medium. Following transfection, the cells were incubated with $4 \mathrm{mg} / \mathrm{ml}$ trastuzumab for $24 \mathrm{~h}$ in RPMI-1640 medium supplemented with $2 \mathrm{mM}$ glutamine and $10 \% \mathrm{FBS}$ at $37^{\circ} \mathrm{C}$.

Reverse transcription-quantitative PCR (RT-qPCR). Total RNA was extracted from the ESO26 cells using TRIzol reagent (Invitrogen; Thermo Fisher Scientific, Inc.) according to the manufacturer's protocol. cDNA was then synthesized from $1 \mu \mathrm{g}$ total RNA using a SuperScript ${ }^{\circledR}$ VILO $^{\text {TM }}$ cDNA Synthesis kit (Thermo Fisher Scientific Inc.). The temperature for the reverse transcription reaction was at $42^{\circ} \mathrm{C}$. Primers used for PCR were: SOX9 forward, 5'-ttgagccttaaaacggtgct-3' and reverse, 5'-tggtgttctgagaggcacag-3'; $\beta$-actin forward, 5'-ggacttcgagcaagagatgg-3' and reverse, 5'-agcactgtgttggcgtacag-3'. The qPCR was performed using the LightCycler 480 Instrument II (Roche Diagnostics, Basel, Switzerland) using LightCycler 480 SYBR-Green I Master (Roche Diagnostics) in accordance with the manufacturer's instructions. Thermocycler conditions were: $95^{\circ} \mathrm{C}$ for $10 \mathrm{~min}$, followed by 40 cycles of $95^{\circ} \mathrm{C}$ for $15 \mathrm{sec}, 60^{\circ} \mathrm{C}$ for $30 \mathrm{sec}$, and $72^{\circ} \mathrm{C}$ for $1 \mathrm{~min}$. All reactions were performed in triplicate. The expression of SOX9 was normalized against the $\beta$-actin reference gene using the $2^{-\Delta \Delta \mathrm{Cq}}$ method (18).

Western blot analysis. The ESO26 cells were harvested and lysed with radioimmunoprecipitation assay lysis buffer (Beyotime Institute of Biotechnology, Shanghai, China) at room temperature for $5 \mathrm{sec}$, and then were centrifuged at $13,000 \times \mathrm{g}$ for $5 \mathrm{~min}$ at room temperature. Protein concentration was quantified using a Bradford Protein Assay kit (Beyotime Institute of Biotechnology). A total of $50 \mu \mathrm{g}$ protein per lane was separated by $10 \%$ SDS-PAGE, and then transferred onto polyvinylidene fluoride membranes (Bio-Rad Laboratories, Inc., Hercules, CA, USA). The membranes were blocked by incubation with $5 \%$ non-fat milk at $4{ }^{\circ} \mathrm{C}$ overnight, and washed with Tris-buffered saline with Tween-20 (TBST) three times. Subsequently, the membranes were incubated with the following primary antibodies: SOX9 rabbit polyclonal antibody (ab26414; 1:400 dilution; Abcam, Cambridge, MA, USA), phospho-AKT (Ser473) rabbit polyclonal antibody (cat. no. 9271; 1:800 dilution; Cell Signaling Technology, Inc., Beverly, MA, USA), AKT mouse monoclonal antibody (cat. no. 2967; 1:1,000 dilution; Cell Signaling Technology, Inc.) and GAPDH mouse monoclonal antibody (ab8245; 1:1,000 dilution; Abcam) at $4^{\circ} \mathrm{C}$ overnight. After washing with TBST, the membranes were incubated with horseradish peroxidase (HRP)-labeled rabbit anti-mouse IgG (ab6728; 1:2,000 dilution; Abcam) or HRP-labeled goat anti-mouse IgG (ab6789; 1:2,000 dilution; Abcam) at $37^{\circ} \mathrm{C}$ for $2 \mathrm{~h}$. The protein bands were visualized using an ECL Western Blotting Detection kit (Pierce; Thermo Fisher Scientific, Inc.). Protein levels were analyzed using densitometric analysis using ImageJ software version 1.46 (National Institutes of Health; Bethesda, MD, USA). The experiment was performed in triplicate.

MTT assay. MTT assay was used to investigate the proliferation of the transfected ESO26 cells with and without trastuzumab treatment. The cells were incubated in 96-well plates at a density of $3 \times 10^{3}$ cells per well, and were exposed to $4 \mathrm{mg} / \mathrm{ml}$ trastuzumab for $24,48,72$ and $96 \mathrm{~h}$. MTT solution (Sigma-Aldrich; Merck KGaA, Darmstadt, Germany) was then added to each well, and the cells were incubated at $37^{\circ} \mathrm{C}$ for $4 \mathrm{~h}$. Medium with no cells was used as the blank control. Subsequently, $150 \mu 1$ dimethyl sulfoxide (Sigma-Aldrich; Merck $\mathrm{KGaA}$ ) was added to each well to dissolve the formazan crystals. The optical density was measured at a wavelength of $490 \mathrm{~nm}$ using a microplate reader (ELx800; BioTek Instruments, Inc., Winooski, VT, USA).

Flow cytometry (FCM). Cell apoptosis of the transfected ESO26 cells with and without trastuzumab treatment was determined using an Annexin V-fluorescein isothiocyanate (FITC) Apoptosis Detection kit (BioVision Inc., Milpitas, CA, USA). The cells were collected and washed with PBS. They were then resuspended in Binding Buffer, and incubated with $5 \mu \mathrm{l}$ Annexin V-FITC and $10 \mu \mathrm{l}$ propidium iodide (PI) at room temperature for $15 \mathrm{~min}$ in the dark. Subsequently, $400 \mu \mathrm{l}$ Binding Buffer were added. The stained cells were detected and cell apoptosis rates were determined using a flow cytometer (FACSCalibur; BD Biosciences, San Jose, CA, USA) with CellQuest Pro software (BD Biosciences). 
A

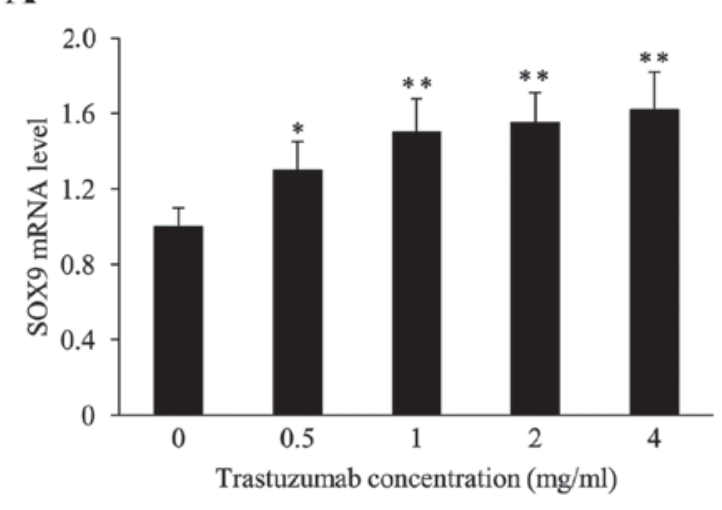

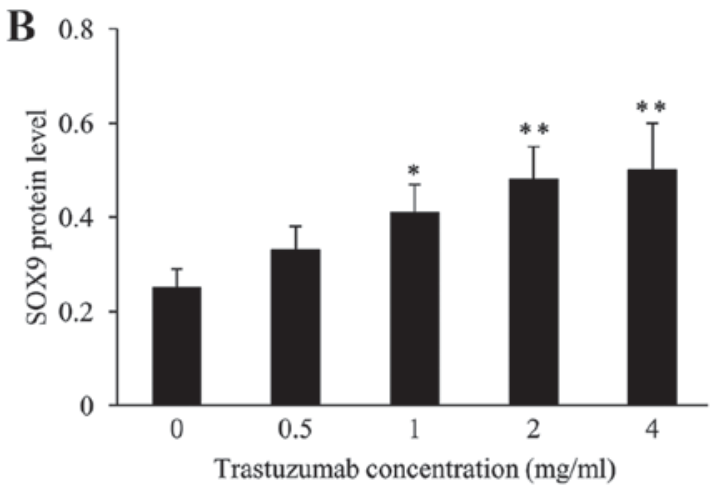

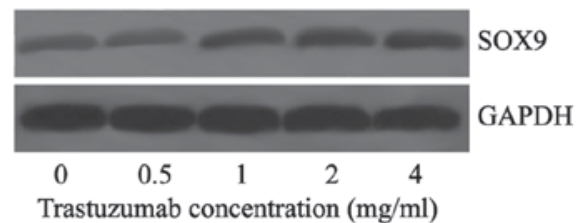

Figure 1. Expression of SOX9 in ESO26 cells following treatment with various concentrations of trastuzumab. Relative (A) mRNA and (B) protein levels of SOX9 in cells treated with $0,0.5,1,2$ and $4 \mathrm{ng} / \mathrm{ml}$ trastuzumab. Data are presented as the mean + standard deviation ${ }^{*} \mathrm{P}<0.05$ and ${ }^{* *} \mathrm{P}<0.01 \mathrm{vs} .0 \mathrm{mg} / \mathrm{ml} . \mathrm{SOX} 9$, sex determining region Y-box 9.

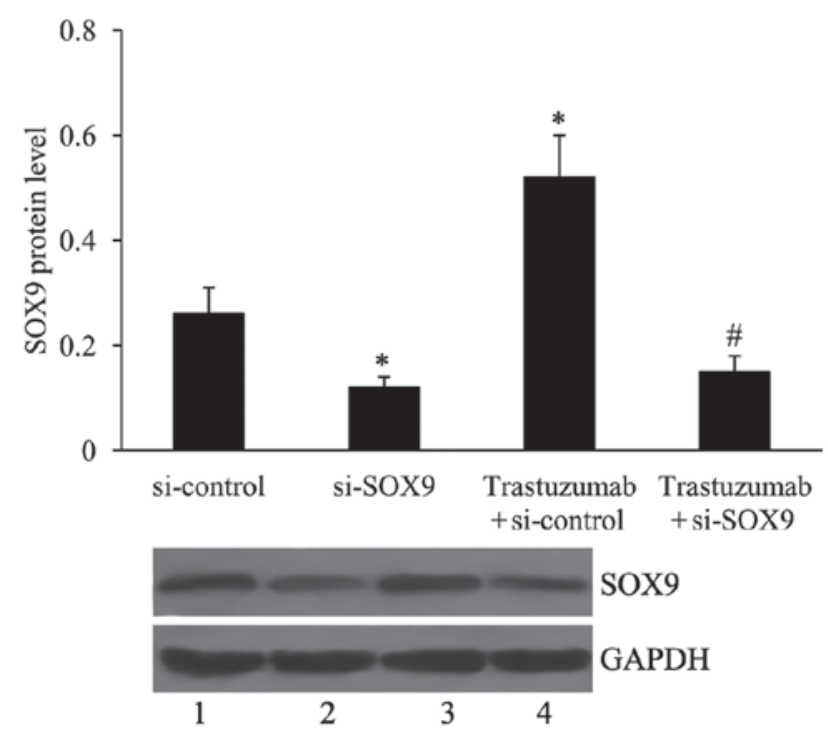

Figure 2. Protein expression of SOX9 in si-control or si-SOX9-transfected cells with or without trastuzumab treatment. Lane 1, si-control; lane 2, si-SOX9; lane 3, trastuzumab + si-control; lane 4, trastuzumab + si-SOX9. ${ }^{*} \mathrm{P}<0.01$ vs. the si-control group; ${ }^{\#} \mathrm{P}<0.01$ vs. the trastuzumab + si-control group. SOX9, sex determining region Y-box 9; si, small interfering RNA.

Statistical analysis. Statistical analysis was performed using SPSS 19.0 statistical analysis software (IBM Corp., Armonk, NY, USA). Data are presented as the mean \pm or + standard deviation. Statistical significance for experiments was determined by one-way analysis of variance followed by the least significant difference test. $\mathrm{P}<0.05$ was to indicate a statistically significant difference.

\section{Results}

Effect of trastuzumab on SOX9 expression in ESO26 cells. To investigate the effect of trastuzumab on SOX9 expression, ESO26 cells were treated with various concentrations of trastuzumab for $24 \mathrm{~h}$, and then SOX9 expression was determined using RT-qPCR and western blotting. The mRNA and protein levels of SOX9 were significantly upregulated in ESO26 cells treated with 1,2 or $4 \mathrm{mg} / \mathrm{ml}$ trastuzumab compared with those in the untreated ESO26 cells (Fig. 1).

Effect of si-SOX9 on SOX9 expression in ESO26 cells. ESO26 cells were transfected with si-SOX9, and then incubated with $4 \mathrm{mg} / \mathrm{ml}$ trastuzumab for $24 \mathrm{~h}$. Subsequently, SOX9 expression was examined using western blotting. The SOX9 protein level was significantly downregulated in the si-SOX9-transfected cells compared with the si-control cells in the presence or absence of trastuzumab (Fig. 2).

Effect of si-SOX9 on ESO26 cell proliferation. Cell proliferation following transfection with si-control or si-SOX9 was determined using an MTT assay. As shown in Fig. 3 the proliferation of the cells transfected with the si-control was significantly suppressed by trastuzumab treatment. In the SOX9 knockdown cells, proliferation was also significantly inhibited by trastuzumab.

Effect of si-SOX9 on ESO26 cell apoptosis. The effect of si-SOX9 on ESO26 cell apoptosis was examined using FCM analysis. The results revealed that compared with the cells in the si-control group, the number of apoptotic cells was significantly increased by transfection with si-SOX 9 and by treatment with trastuzumab. In the presence of trastuzumab, the number of apoptotic cells was greater in the SOX9 knockdown cells compared with the si-control cells (Fig. 4).

Effect of si-SOX9 on the PI3K/AKT pathway in ESO26 cells. To investigate the effect of si-SOX9 on PI3K/AKT activation, the expression of phosphorylated AKT was measured. As shown in Fig. 5, trastuzumab treatment activated the PI3K/AKT signaling pathway, as demonstrated by an elevated level of phosphorylated AKT in the trastuzumab-treated cells 


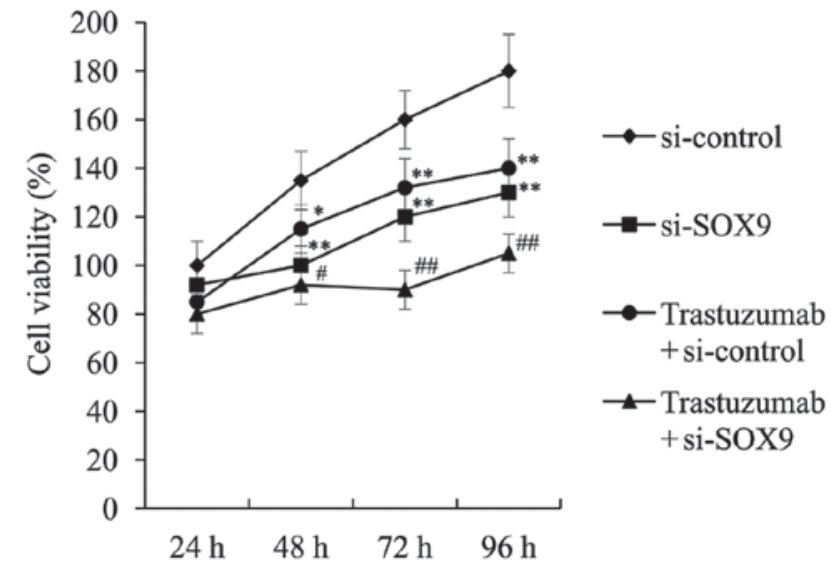

Figure 3. Cell proliferation of si-control or si-SOX9-transfected cells with or without trastuzumab treatment. ${ }^{*} \mathrm{P}<0.05$ and ${ }^{* * *} \mathrm{P}<0.01$ vs. the si-control group; ${ }^{\#} \mathrm{P}<0.05$ and ${ }^{\# \#} \mathrm{P}<0.01$ compared with trastuzumab + si-control group. SOX9, sex determining region Y-box 9; si, small interfering RNA.

compared with the respective untreated cells. Si-SOX9 transfection did not exhibit any effects on PI3K/AKT signaling activation in untreated ESO26 cells; however, the level of phosphorylated AKT was significantly decreased by si-SOX9 in trastuzumab-treated cells.

\section{Discussion}

In the present study, it was demonstrated that trastuzumab induced SOX9 expression in a concentration-dependent manner. SOX9 knockdown sensitized ESO26 cells to trastuzumab by inhibiting cell proliferation and increasing cell apoptosis. Furthermore, the trastuzumab-induced activation of PI3K/AKT signaling was suppressed by SOX9 knockdown.

SOX9, plays vital roles in embryonic development (19). The importance of SOX9 in tumorigenesis has been identified, and the dysregulation of SOX9 has been implicated in several cancers, including prostate cancer, lung adenocarcinoma and gastric carcinoma (20-22). Overexpression of SOX9 has been demonstrated to increase tumor growth, invasion and angiogenesis $(14,23)$. In the present study, it was revealed that SOX9 knockdown led to suppressed cell proliferation and increased cell apoptosis, indicating that SOX9 is a tumor promoter in AEG. This finding is consistent with the role of SOX9 in other types of tumor (20-22). It has previously been reported that SOX9 mediates the effect of certain antitumor drugs; SOX9 expression was markedly inhibited in mice tumors by combination treatment with ABT-263 and 5-FU (24). In renal cell carcinoma, SOX9 was demonstrated to be involved in resistance to tyrosine kinase inhibitors (TKIs) and indicated to be a promising biomarker predicting the response to TKI treatment; patients who were SOX9 negative exhibited a better response to TKI treatment than did those who were SOX9 positive (25). The present study firstly demonstrated that SOX9 is involved in the resistance of AEG to trastuzumab. SOX9 expression was shown to be induced by trastuzumab in a concentration-dependent manner in ESO26 cells. In addition, the trastuzumab treatment suppressed cell proliferation and promoted the apoptosis of ESO26 cells, and these effects were further increased by transfection with si-SOX9. These

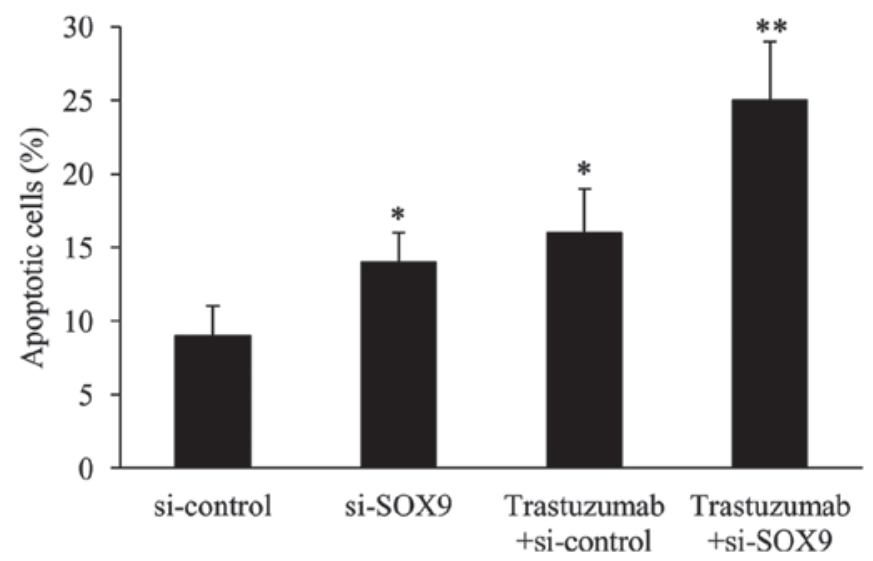

Figure 4. Cell apoptosis rate of si-control or si-SOX9-transfected cells with or without trastuzumab treatment. ${ }^{*} \mathrm{P}<0.05$ vs. the si-control group; ${ }^{* *} \mathrm{P}<0.01$ vs. the trastuzumab + si-control group. SOX9, sex determining region Y-box 9; si, small interfering RNA.
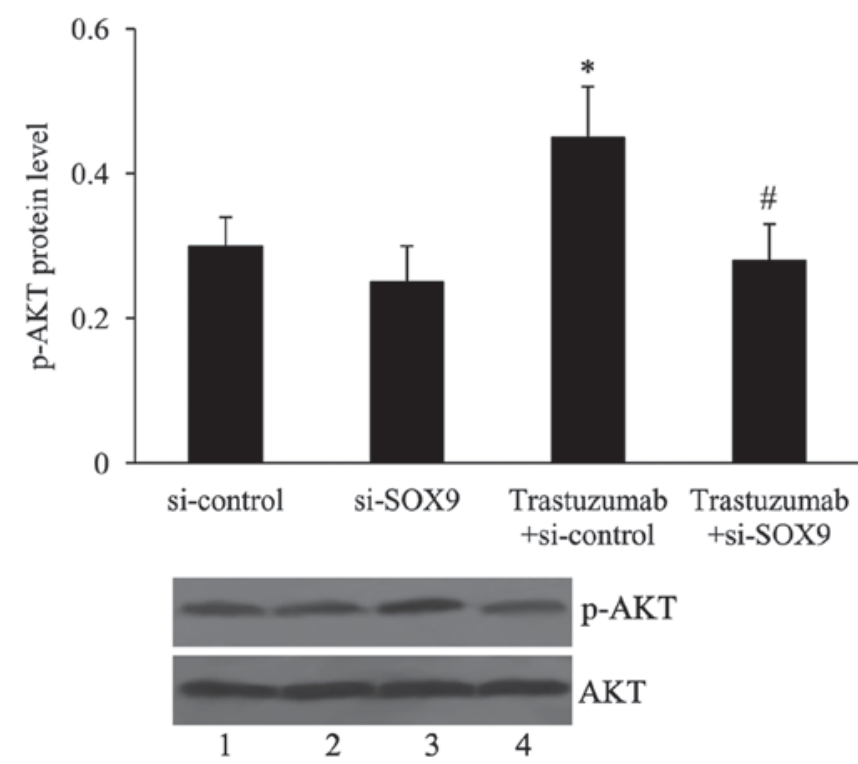

Figure 5. Expression of p-AKT in si-control or si-SOX9-transfected cells with or without trastuzumab treatment. Lane 1, si-control; lane 2, si-SOX9; lane 3, trastuzumab + si-control; lane 4 , trastuzumab + si-SOX9. ${ }^{*} \mathrm{P}<0.05$ vs. the si-control group; ${ }^{\#} \mathrm{P}<0.01$ vs. the trastuzumab + si-control group. p, phosphorylated; SOX9, sex determining region Y-box 9; si, small interfering RNA.

findings suggested that SOX9 promotes the resistance of AEG cells to trastuzumab.

The PI3K/AKT signaling pathway is an intracellular signaling pathway important in regulating cell proliferation, cell cycle, apoptosis and differentiation (26-28). PI3K activation leads to phosphorylation and activation of the serine/threonine kinase AKT (29); therefore, AKT phosphorylation may be used to assess the activity of the PI3K/AKT signaling pathway. Aberrant PI3K/AKT signaling has been detected in several cancer cell lines, and it has been reported that activation of the PI3K/AKT signaling pathway may predict the resistance to chemotherapy and molecularly targeted therapy (30-32). Recently, Liu et al (16) and Zuo et al (17) reported that activation of the PI3K/AKT signaling pathway leads to the resistance of gastric cancer to trastuzumab. To investigate whether the 
activity of the PI3K/AKT signaling pathway was affected by SOX9, si-SOX9 was used to transfect ESO26 cells in the present study. It was found that the trastuzumab-induced phosphorylation of AKT was suppressed by SOX9 knockdown. These results demonstrated that SOX 9 mediates the effect of trastuzumab on PI3K/AKT signaling activation.

A limitation of the present study is that only one cell line, ESO26, was used to investigate the role of SOX9 in trastuzumab resistance in AEG. Further studies using additional cell lines are required to support the results.

In conclusion, the present study demonstrated that SOX9 participated in trastuzumab resistance by affecting cell proliferation and apoptosis. In addition, SOX9 affected trastuzumab-induced PI3K/AKT signaling activation in AEG. The present study provided in vitro evidence of the role of SOX9 in the resistance of AEG to trastuzumab. These issues require further elucidation in clinical studies.

\section{Acknowledgements}

The present study was supported by the Shenzhen Science and Technology Project (grant no. JCYJ20150403101028177).

\section{References}

1. Pohl H and Welch HG: The role of overdiagnosis and reclassification in the marked increase of esophageal adenocarcinoma incidence. J Natl Cancer Inst 97: 142-146, 2005.

2. Rüdiger Siewert J, Feith M, Werner $M$ and Stein HJ: Adenocarcinoma of the esophagogastric junction: Results of surgical therapy based on anatomical/topographic classification in 1,002 consecutive patients. Ann Surg 232: 353-361, 2000.

3. Botterweck AA, Schouten LJ, Volovics A, Dorant E and van Den Brandt PA: Trends in incidence of adenocarcinoma of the oesophagus and gastric cardia in ten European countries. Int J Epidemiol 29: 645-654, 2000.

4. Edgren G, Adami HO, Weiderpass E and Nyrén O: A global assessment of the oesophageal adenocarcinoma epidemic. Gut 62: 1406-1414, 2013.

5. Dikken JL, Lemmens VE, Wouters MW, Wijnhoven BP, Siersema PD, Nieuwenhuijzen GA, van Sandick JW, Cats A, Verheij M, Coebergh JW and van de Velde CJ: Increased incidence and survival for oesophageal cancer but not for gastric cardia cancer in the Netherlands. Eur J Cancer 48: 1624-1632, 2012.

6. Brown LM, Devesa SS and Chow WH: Incidence of adenocarcinoma of the esophagus among white Americans by sex, stage, and age. J Natl Cancer Inst 100: 1184-1187, 2008.

7. de Jonge PJ, van Blankenstein M, Grady WM and Kuipers EJ: Barrett's oesophagus: Epidemiology, cancer risk and implications for management. Gut 63: 191-202, 2014.

8. Siewert JR, Stein HJ and Feith M: Adenocarcinoma of the esophago-gastric junction. Scand J Surg 95: 260-269, 2006.

9. Bang YJ, Van Cutsem E, Feyereislova A, Chung HC, Shen L, Sawaki A, Lordick F, Ohtsu A, Omuro Y, Satoh T, et al: Trastuzumab in combination with chemotherapy versus chemotherapy alone for treatment of HER2-positive advanced gastric or gastro-oesophageal junction cancer (ToGA): A phase 3, open-label, randomised controlled trial. Lancet 376: 687-697, 2010.

10. Strong VE, D'Amico TA, Kleinberg L and Ajani J: Impact of the 7th Edition AJCC staging classification on the NCCN clinical practice guidelines in oncology for gastric and esophageal cancers. J Natl Compr Canc Netw 11: 60-66, 2013.

11. Ma F, Ye H, He HH, Gerrin SJ, Chen S, Tanenbaum BA, Cai C, Sowalsky AG, He L, Wang H, et al: SOX9 drives WNT pathway activation in prostate cancer. J Clin Invest 126: 1745-1758, 2016.

12. Marcker Espersen ML, Linnemann D, Christensen IJ, Alamili M, Troelsen JT and Høgdall E: SOX9 expression predicts relapse of stage II colon cancer patients. Hum Pathol 52: 38-46, 2016.
13. Wang X, Ju Y, Zhou MI, Liu X and Zhou C: Upregulation of SOX 9 promotes cell proliferation, migration and invasion in lung adenocarcinoma. Oncol Lett 10: 990-994, 2015.

14. Chakravarty G, Moroz K, Makridakis NM, Lloyd SA, Galvez SE, Canavello PR, Lacey MR, Agrawal K and Mondal D: Prognostic significance of cytoplasmic SOX9 in invasive ductal carcinoma and metastatic breast cancer. Exp Biol Med (Maywood) 236: 145-155, 2011.

15. Riemenschnitter C, Teleki I, Tischler V, Guo W and Varga Z: Stability and prognostic value of Slug, Sox 9 and Sox 10 expression in breast cancers treated with neoadjuvant chemotherapy. Springerplus 2: 695, 2013.

16. Liu J, Pan C, Guo L, Wu M, Guo J, Peng S, Wu Q and Zuo Q: A new mechanism of trastuzumab resistance in gastric cancer: MACC1 promotes the Warburg effect via activation of the PI3K/AKT signaling pathway. J Hematol Oncol 9: 76, 2016.

17. Zuo Q, Liu J, Zhang J, Wu M, Guo L and Liao W: Development of trastuzumab-resistant human gastric carcinoma cell lines and mechanisms of drug resistance. Sci Rep 5: 11634, 2015.

18. Livak KJ and Schmittgen TD: Analysis of relative gene expression data using real-time quantitative PCR and the 2(-Delta Delta C(T)) method. Methods 25: 402-408, 2001.

19. Jo A, Denduluri S, Zhang B, Wang Z, Yin L, Yan Z, Kang R, Shi LL, Mok J, Lee MJ and Haydon R: The versatile functions of Sox9 in development, stem cells, and human diseases. Genes Dis 1: 149-161, 2014

20. Wang H, McKnight NC, Zhang T, Lu ML, Balk SP and Yuan X: SOX9 is expressed in normal prostate basal cells and regulates androgen receptor expression in prostate cancer cells. Cancer Res 67: 528-536, 2007.

21. Jiang SS, Fang WT, Hou YH, Huang SF, Yen BL, Chang JL, Li SM, Liu HP, Liu YL, Huang CT, et al: Upregulation of SOX9 in lung adenocarcinoma and its involvement in the regulation of cell growth and tumorigenicity. Clin Cancer Res 16: 4363-4373, 2010.

22. Zhou CJ, Guo JQ, Zhu KX, Zhang QH, Pan CR, Xu WH, Wang HJ and Liu B: Elevated expression of SOX9 is related with the progression of gastric carcinoma. Diagn Cytopathol 39: 105-109, 2011.

23. Wang H, Leav I, Ibaragi S, Wegner M, Hu GF, Lu ML, Balk SP and Yuan X: SOX9 is expressed in human fetal prostate epithelium and enhances prostate cancer invasion. Cancer Res 68 : 1625-1630, 2008.

24. Chen Q, Song S, Wei S, Liu B, Honjo S, Scott A, Jin J, Ma L, Zhu H, Skinner HD, et al: ABT-263 induces apoptosis and synergizes with chemotherapy by targeting stemness pathways in esophageal cancer. Oncotarget 6: 25883-25896, 2015.

25. Li XL, Chen XQ, Zhang MN, Chen N, Nie L, Xu M, Gong J, Shen PF, Su ZZ, Weng X, et al: SOX9 was involved in TKIs resistance in renal cell carcinoma via Raf/MEK/ERK signaling pathway. Int J Clin Exp Pathol 8: 3871-3881, 2015.

26. Yu JS and Cui W: Proliferation, survival and metabolism: The role of PI3K/AKT/mTOR signalling in pluripotency and cell fate determination. Development 143: 3050-3060, 2016.

27. Ma Y, Qin H and Cui Y: MiR-34a targets GAS1 to promote cell proliferation and inhibit apoptosis in papillary thyroid carcinoma via PI3K/Akt/Bad pathway. Biochem Biophys Res Commun 441: 958-963, 2013.

28. Wang Y, Wang WL, Xie WL, Li LZ, Sun J, Sun WJ and Gong HY: Puerarin stimulates proliferation and differentiation and protects against cell death in human osteoblastic MG-63 cells via ER-dependent MEK/ERK and PI3K/Akt activation. Phytomedicine 20: 787-796, 2013.

29. Franke TF, Yang SI, Chan TO, Datta K, Kazlauskas A, Morrison DK, Kaplan DR and Tsichlis PN: The protein kinase encoded by the Akt proto-oncogene is a target of the PDGF-activated phosphatidylinositol 3-kinase. Cell 81: 727-736, 1995.

30. Mandell JW: Phosphorylation state-specific antibodies: Applications in investigative and diagnostic pathology. Am J Pathol 163: 1687-1698, 2003.

31. Mandell JW: Immunohistochemical assessment of protein phosphorylation state: The dream and the reality. Histochem Cell Biol 130: 465-471, 2008.

32. Slomovitz BM and Coleman RL: The PI3K/AKT/mTOR pathway as a therapeutic target in endometrial cancer. Clin Cancer Res 18: 5856-5864, 2012. 